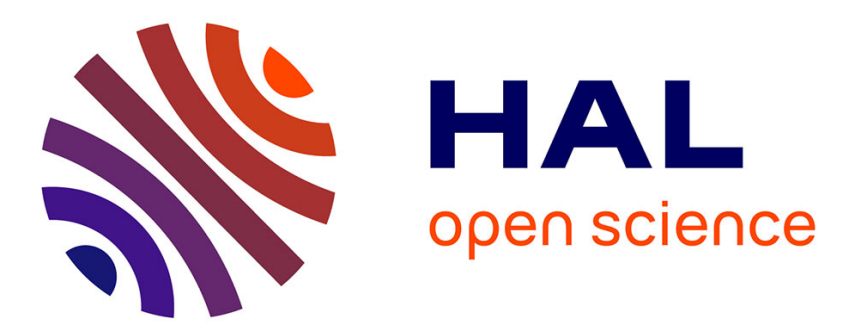

\title{
Enveloping skewfields of the nilpotent positive part and the Borel subsuperalgebra of osp $(1,2 \mathrm{n})$
}

\author{
Jacques Alev, François Dumas
}

\section{To cite this version:}

Jacques Alev, François Dumas. Enveloping skewfields of the nilpotent positive part and the Borel subsuperalgebra of osp(1,2n). Contemporary mathematics, 2019, Rings, Modules and Codes Fifth International Conference Noncommutative Rings and their Applications June 12-15, 2017 University of Artois, Lens, France, 727, pp.7-23. 10.1090/conm/727/14621 . hal-02401624

\section{HAL Id: hal-02401624 \\ https://hal.science/hal-02401624}

Submitted on 10 Dec 2019

HAL is a multi-disciplinary open access archive for the deposit and dissemination of scientific research documents, whether they are published or not. The documents may come from teaching and research institutions in France or abroad, or from public or private research centers.
L'archive ouverte pluridisciplinaire HAL, est destinée au dépôt et à la diffusion de documents scientifiques de niveau recherche, publiés ou non, émanant des établissements d'enseignement et de recherche français ou étrangers, des laboratoires publics ou privés. 


\title{
ENVELOPING SKEWFIELDS OF THE NILPOTENT POSITIVE PART AND THE BOREL SUBSUPERALGEBRA OF osp $(1,2 n)$
}

\author{
JACQUES ALEV AND FRANÇOIS DUMAS
}

\begin{abstract}
We study an analogue of the Gelfand-Kirillov property for some Lie superalgebras. More precisely, we consider in the classical simple orthosymplectic Lie superalgebra $\mathfrak{o s p}(1,2 n)$ of dimension $n^{2}+3 n$ the positive nilpotent subsuperalgebra $\mathfrak{n}^{+}$of dimension $n^{2}+n$ and the solvable Borel subsuperalgebra $\mathfrak{b}^{+}$of dimension $n^{2}+2 n$. We prove that the enveloping algebras $\mathcal{U}\left(\mathfrak{n}^{+}\right)$and $\mathcal{U}\left(\mathfrak{b}^{+}\right)$are rationally equivalent to some super-analogues of Weyl algebras over polynomial superalgebras.
\end{abstract}

\section{INTRODUCTION}

In the classical context of the seminal paper [7], an algebraic finitedimensional complex Lie algebra $\mathfrak{g}$ is said to satisfy the Gelfand-Kirillov property when its enveloping algebra $\mathcal{U}(\mathfrak{g})$ is rationaly equivalent to a Weyl algebra $A_{n}(K)$ over a commutative purely transcendental field extension $K$ of $\mathbb{C}$. It was proved that this property is satisfied when $\mathfrak{g}$ is nilpotent ([7]), or more generally solvable ([5], [10], [12]). This profound problem gave rise to an abundant literature with many developments within Lie theory itself (see references in [3] or [16]) or for variants concerning quantum groups, invariant algebras or other classes of interesting noncommutatives algebras.

The study of an analogue of the Gelfand-Kirillov property for Lie superalgebras was explored in [13] and [2]. In this context, it is relevant to introduce for any integer $n \geq 1$ the noncommutative polynomial superalgebra $\widetilde{\mathcal{O}}\left(\mathbb{C}^{n}\right)$ and an analogue $\widetilde{A}_{n}(\mathbb{C})$ of the Weyl algebra. By definition $\widetilde{\mathcal{O}}\left(\mathbb{C}^{n}\right)$ is the algebra generated over $\mathbb{C}$ by $n$ indeterminates $y_{1}, \ldots, y_{n}$ with relations $y_{i} y_{j}+y_{j} y_{i}=0$ for all $1 \leq i \neq j \leq n$. Then $\widetilde{A}_{n}(\mathbb{C})$ is the algebra generated over $\widetilde{\mathcal{O}}\left(\mathbb{C}^{n}\right)$ by $n$ other indeterminates $x_{1}, \ldots, x_{n}$ with relations $x_{i} y_{j}+y_{j} x_{i}=x_{i} x_{j}+x_{j} x_{i}=0$ for all $1 \leq i \neq j \leq n$ and $x_{i} y_{i}-y_{i} x_{i}=1$ for any $1 \leq i \leq n$. These algebras are noetherian domains and then they admit a skewfield of fractions. Otherwise the only case in the classification of classical simple finite dimensional complex Lie superalgebras $\mathfrak{g}$ where the

Date: April 23, 2018.

2010 Mathematics Subject Classification. Primary 17B35; Secondary 16S30, 16S85, $16 \mathrm{~K} 40$.

Key words and phrases. Simple Lie superalgebra, enveloping algebra, Gelfand-Kirillov hypothesis, Weyl algebra, skewfield. 
enveloping algebra $\mathcal{U}(\mathfrak{g})$ is a domain is when $\mathfrak{g}$ is an orthosymplectic Lie superalgebra $\mathfrak{o s p}(1,2 n)$ (see [4, 9]). This is the case we consider here.

For any integer $n \geq 1$, the superalgebra $\mathfrak{g}=\mathfrak{o} \mathfrak{s p}(1,2 n)$ is of dimension $2 n^{2}+3 n$. In its $\mathbb{Z}_{2}$-graded decomposition $\mathfrak{g}=\mathfrak{g}_{\overline{0}} \oplus \mathfrak{g}_{\overline{1}}$, the even part $\mathfrak{g}_{\overline{0}}$ is isomorphic to the symplectic Lie algebra $\mathfrak{s p}(2 n)$ of dimension $2 n^{2}+n$, and the odd part $\mathfrak{g}_{\overline{1}}$ is a vector space of dimension $2 n$. There is a natural definition of the nilpotent positive part $\mathfrak{n}^{+}=\mathfrak{o s p}^{+}(1,2 n)$, which is a nilpotent subsuperalgebra of $\mathfrak{g}$ of dimension $n^{2}+n$. In its $\mathbb{Z}_{2}$-graded decomposition $\mathfrak{n}^{+}=\mathfrak{n}_{\overline{0}}^{+} \oplus \mathfrak{n}_{\overline{1}}^{+}$, the even part $\mathfrak{n}_{\overline{0}}^{+} \simeq \mathfrak{s p}^{+}(2 n)$ is just the nilpotent positive part of dimension $n^{2}$ in the ordinary triangular decomposition of the Lie algebra $\mathfrak{g}_{\overline{0}} \simeq \mathfrak{s p}(2 n)$, and the odd part $\mathfrak{n}_{1}^{+}$is a subspace of dimension $n$ in $\mathfrak{g}_{\overline{1}}$. Denoting by $\mathfrak{h}$ the Cartan Lie subalgebra of $\mathfrak{g}_{\overline{0}}$, the Borel subsuperalgebra $\mathfrak{b}^{+}$of $\mathfrak{g}$ is defined as the subsuperalgebra generated by $\mathfrak{n}^{+}$and $\mathfrak{h}$. We have $\mathfrak{b}^{+}=\mathfrak{n}^{+} \oplus \mathfrak{h}=\mathfrak{b}_{\overline{0}}^{+} \oplus \mathfrak{n}_{\overline{1}}^{+}$, where the even part $\mathfrak{b}_{\overline{0}}^{+}=\mathfrak{n}_{\overline{0}}^{+} \oplus \mathfrak{h}$ is the positive Borel Lie subalgebra of $\mathfrak{g}_{\overline{0}} \simeq \mathfrak{s p}(2 n)$. In particular, $\mathfrak{b}_{\overline{0}}^{+}$and $\mathfrak{b}^{+}$are of dimensions $n^{2}+n$ and $n^{2}+2 n$ respectively. The enveloping algebras $\mathcal{U}\left(\mathfrak{n}^{+}\right)$and $\mathcal{U}\left(\mathfrak{b}^{+}\right)$ can be described as iterated skew polynomial algebras; then they are noetherian domains and therefore we can consider their skewfields of fractions. We prove in the paper:

Main theorem. For any $n \geq 2$, the enveloping algebras of the subsuperalgebras $\mathfrak{n}^{+}$and $\mathfrak{b}^{+}$of $\mathfrak{o s p}(1,2 n)$ satisfy the following isomorphisms:

(i) $\operatorname{Frac} \mathcal{U}\left(\mathfrak{n}^{+}\right) \simeq \operatorname{Frac}\left(A_{n(n-1) / 2}(\mathbb{C}) \otimes \widetilde{\mathcal{O}}\left(\mathbb{C}^{n}\right)\right)$, and its center is a purely transcendental extension of dimension $n$ over $\mathbb{C}$.

(ii) $\operatorname{Frac} \mathcal{U}\left(\mathfrak{b}^{+}\right) \simeq \operatorname{Frac}\left(A_{n(n-1) / 2}(\mathbb{C}) \otimes \widetilde{A}_{n}(\mathbb{C})\right)$, and its center is $\mathbb{C}$.

The method we use to prove this theorem allows to recover as a corollary the classical Gelfand-Kirillov property for the even parts, with already known descriptions of Frac $\mathcal{U}\left(\mathfrak{n}_{0}^{+}\right)$as a Weyl skewfield $D_{n(n-1) / 2}(K)$ over a commutative field $K$ which is a purely transcendental extension of dimension $n$ over $\mathbb{C}$, and of Frac $\mathcal{U}\left(\mathfrak{b}_{\overline{0}}^{+}\right)$as a Weyl skewfield $D_{n(n+1) / 2}(\mathbb{C})$ over $\mathbb{C}$. The particular case $n=2$ of the main theorem was previously proved in Proposition 3.2 and Theorem 3.4 of [2]. The natural and probably difficult question of an extension of the main theorem to the enveloping algebra of the Lie superalgebra $\mathfrak{o s p}(1,2 n)$ itself must be appreciated by recalling that even for the even part the answer is unknown, the case of $\mathfrak{s p}(2 n)$ being one of the situations where the original Gelfand-Kirillov conjecture remains open (see [16]).

The paper is in three parts. The first one introduces the analogues of Weyl algebras for polynomial superalgebras and some canonical form of their skewfields of fractions. The second part describes the enveloping algebras $\mathcal{U}\left(\mathfrak{n}^{+}\right)$and $\mathcal{U}\left(\mathfrak{b}^{+}\right)$as iterated skew polynomial algebras. The last section contains the proof of the main theorem, based on suitable rational changes of generators reducing step by step the commutation relations. 


\section{Analogues of Weyl algebras for polynomial superalgebras}

\subsection{Polynomial superalgebras.}

1.1.1. Notations. It is usual in quantum group theory to denote by $\mathcal{O}_{\Lambda}\left(\mathbb{C}^{n}\right)$ the algebra of polynomials in $n$ indeterminates $y_{1}, \ldots, y_{n}$ with coefficients in $\mathbb{C}$ and noncommutative multiplication twisted by relations $y_{i} y_{j}=\lambda_{i j} y_{j} y_{i}$ for any $1 \leq i, j \leq n$, where $\Lambda=\left(\lambda_{i j}\right)$ is a $n \times n$ multiplicatively skew-symmetric matrix with entries in $\mathbb{C}$. In the particular cases where all $\lambda_{i j}=1$, or $\lambda_{i j}=-1$ for all $i \neq j$, we use respectively the two following notations:

(i) $\mathcal{O}\left(\mathbb{C}^{n}\right)$ is the commutative algebra of polynomials in $n$ indeterminates $y_{1}, y_{2}, \ldots, y_{n}$ with coefficients in $\mathbb{C}$.

(ii) $\widetilde{\mathcal{O}}\left(\mathbb{C}^{n}\right)$ is the noncommutative algebra of polynomials in $n$ indeterminates $y_{1}, y_{2}, \ldots, y_{n}$ with coefficients in $\mathbb{C}$ and product twisted by relations $y_{i} y_{j}=-y_{j} y_{i}$ for any $1 \leq i \neq j \leq n$.

1.1.2. Superalgebra structure. The monomials $y_{1}{ }^{\beta_{1}} y_{2}{ }^{\beta_{2}} \cdots y_{n}{ }^{\beta_{n}}$ whose total degree $\beta_{1}+\beta_{2}+\cdots+\beta_{n}$ is even generate a commutative subalgebra $R_{0}$ of $\widetilde{\mathcal{O}}\left(\mathbb{C}^{n}\right)$. Denoting by $R_{1}$ the $R_{0}$-submodule of $\widetilde{\mathcal{O}}\left(\mathbb{C}^{n}\right)$ generated by $y_{1}, \ldots, y_{n}$, the $\mathbb{C}$-submodules $R_{0}$ and $R_{1}$ satisfy $\widetilde{\mathcal{O}}\left(\mathbb{C}^{n}\right)=R_{0} \oplus R_{1}$ and $R_{i} R_{j} \subseteq R_{i+j}$ (with indices taken modulo 2). This gives rise to a structure of superalgebra on $\widetilde{\mathcal{O}}\left(\mathbb{C}^{n}\right)$. The algebras $\widetilde{\mathcal{O}}\left(\mathbb{C}^{n}\right)$ being noetherian domains, they admit skew fields of fractions. The following proposition gives an alternative form of $\widetilde{\mathcal{O}}\left(\mathbb{C}^{n}\right)$ in terms of planes $\widetilde{\mathcal{O}}\left(\mathbb{C}^{2}\right)$ up to rational equivalence.

\subsubsection{Proposition. For any integer $n \geq 2$, we have:}

$$
\begin{aligned}
& \text { If } n=2 p, \text { then } \operatorname{Frac}\left(\widetilde{\mathcal{O}}\left(\mathbb{C}^{n}\right)\right) \simeq \operatorname{Frac}\left(\widetilde{\mathcal{O}}\left(\mathbb{C}^{2}\right)^{\otimes p}\right) \\
& \text { If } n=2 p+1, \text { then } \operatorname{Frac}\left(\widetilde{\mathcal{O}}\left(\mathbb{C}^{n}\right)\right) \simeq \operatorname{Frac}\left(\widetilde{\mathcal{O}}\left(\mathbb{C}^{2}\right)^{\otimes p} \otimes \mathcal{O}(\mathbb{C})\right)
\end{aligned}
$$

Proof. Suppose that $n \geq 3$ and define from generators $y_{1}, \ldots, y_{n}$ of $\widetilde{\mathcal{O}}\left(\mathbb{C}^{n}\right)$ with $y_{i} y_{j}=-y_{j} y_{i}$ for all $1 \leq i \neq j \leq n$ the monomials: $y_{1}^{\prime}=y_{1}, y_{2}^{\prime}=y_{2}$ and $y_{i}^{\prime}=y_{1} y_{2} y_{i}$ for any $3 \leq i \leq n$. They satisfy $y_{1}^{\prime} y_{2}^{\prime}=-y_{2}^{\prime} y_{1}^{\prime}$ and

$$
y_{1}^{\prime} y_{i}^{\prime}=y_{i}^{\prime} y_{1}^{\prime}, \quad y_{2}^{\prime} y_{i}^{\prime}=y_{i}^{\prime} y_{2}^{\prime}, \quad y_{i}^{\prime} y_{j}^{\prime}=-y_{j}^{\prime} y_{i}^{\prime} \quad \text { for all } 3 \leq i \neq j \leq n .
$$

It is clear that the subfield of Frac $\widetilde{\mathcal{O}}\left(\mathbb{C}^{n}\right)$ generated by $y_{1}^{\prime}, y_{2}^{\prime}, \ldots, y_{n}^{\prime}$ is equal to $\operatorname{Frac} \widetilde{\mathcal{O}}\left(\mathbb{C}^{n}\right)$. We deduce that $\operatorname{Frac} \widetilde{\mathcal{O}}\left(\mathbb{C}^{n}\right) \simeq \operatorname{Frac}\left(\widetilde{\mathcal{O}}\left(\mathbb{C}^{2}\right) \otimes \widetilde{\mathcal{O}}\left(\mathbb{C}^{n-2}\right)\right)$, and the result follows by iteration.

\subsection{Weyl algebras for polynomial superalgebras.}

1.2.1. Notations. The definition of quantum differential calculi on various quantum algebras with adapted de Rham complexes gives rise to suitable versions of quantum Weyl algebras. In the case of quantum spaces $\mathcal{O}_{\Lambda}\left(\mathbb{C}^{n}\right)$, these algebras $A_{n}^{\bar{q}, \Lambda}$ (introduced in [1] and [19] and studied in many papers) take in consideration both the quantization parameters $\Lambda=\left(\lambda_{i j}\right)$ of the 
space and the quantization parameters $\bar{q}=\left(q_{1}, \ldots, q_{n}\right)$ of the "differential" operators on this space. We are interested here in two particular cases:

(i) For $\lambda_{i j}=q_{i}=1$ for all $1 \leq i, j \leq n$, we find the usual Weyl algebra $A_{n}(\mathbb{C})$, that is the algebra of polynomials in $2 n$ indeterminates $x_{1}, \ldots, x_{n}, y_{1}, \ldots, y_{n}$ with coefficients in $\mathbb{C}$ and a noncommutative product twisted by relations:

$$
\begin{cases}x_{i} x_{j}-x_{j} x_{i}=y_{i} y_{j}-y_{j} y_{i}=x_{i} y_{j}-y_{j} x_{i}=0 & (1 \leq i \neq j \leq n), \\ x_{i} y_{i}-y_{i} x_{i}=1 & (1 \leq i \leq n) .\end{cases}
$$

We also introduce the subalgebra $U_{n}(\mathbb{C})$ of $A_{n}(\mathbb{C})$ generated by $y_{1}, \ldots, y_{n}$ and $w_{1}, \ldots, w_{n}$ with $w_{i}=y_{i} x_{i}$ for any $1 \leq i \leq n$. We have:

$$
\begin{cases}w_{i} w_{j}-w_{j} w_{i}=y_{i} y_{j}-y_{j} y_{i}=w_{i} y_{j}-y_{j} w_{i}=0 & (1 \leq i \neq j \leq n), \\ w_{i} y_{i}-y_{i} w_{i}=y_{i} & (1 \leq i \leq n) .\end{cases}
$$

(ii) For $q_{i}=1$ and $\lambda_{i j}=-1$ for all $1 \leq i \neq j \leq n$, we denote by $\widetilde{A}_{n}(\mathbb{C})$ the algebra of polynomials in $2 n$ indeterminates $x_{1}, \ldots, x_{n}, y_{1}, \ldots, y_{n}$ with coefficients in $\mathbb{C}$ and a noncommutative product twisted by:

$$
\begin{cases}x_{i} x_{j}+x_{j} x_{i}=y_{i} y_{j}+y_{j} y_{i}=x_{i} y_{j}+y_{j} x_{i}=0 & (1 \leq i \neq j \leq n), \\ x_{i} y_{i}-y_{i} x_{i}=1 & (1 \leq i \leq n) .\end{cases}
$$

We denote by $\widetilde{U}_{n}(\mathbb{C})$ the subalgebra of $\widetilde{A}_{n}(\mathbb{C})$ generated by $y_{1}, \ldots, y_{n}$ and $w_{1}, \ldots, w_{n}$ with $w_{i}=y_{i} x_{i}$ for any $1 \leq i \leq n$. We have:

$$
\begin{cases}y_{i} y_{j}+y_{j} y_{i}=0 & (1 \leq i \neq j \leq n), \\ w_{i} w_{j}-w_{j} w_{i}=w_{i} y_{j}-y_{j} w_{i}=0 & (1 \leq i \neq j \leq n), \\ w_{i} y_{i}-y_{i} w_{i}=y_{i} & (1 \leq i \leq n) .\end{cases}
$$

1.2.2. Remarks. The algebras $\widetilde{A}_{n}(\mathbb{C})$ are the algebras denoted by $S_{n, n}^{\Lambda}$ and studied in [17] and [18] when all non diagonal entries of $\Lambda$ are equal to -1 . Section 11 of [19] gives a slightly different presentation of these algebras as "generalized enveloping algebras"; with the notations of this article, case (i) corresponds to the values $q_{i j}=p_{i}=q=1$, and case (ii) is for $q_{i j}=-1$ and $p_{i}=q=1$. They also appear in example 2.1 of [8] (taking $q=1$ and $p_{i j}=-1$ ). We refer to paragraph 1.3.3 of [17] for more ringtheoretical references and a survey, and only mention here that, for any integer $n \geq 1$, the algebra $\widetilde{A}_{n}(\mathbb{C})$ is simple, has center $\mathbb{C}$, and has the same Hochschild homology and cohomology as the usual Weyl algebra $A_{n}(\mathbb{C})$.

\subsubsection{Superalgebra structure.}

(i) Let $\mathfrak{g}_{0}$ and $\mathfrak{g}_{1}$ be two $\mathbb{C}$-vector spaces of dimension $n$ with respective basis $\left\{z_{1}, \ldots, z_{n}\right\}$ and $\left\{y_{1}, \ldots, y_{n}\right\}$. Let $\mathfrak{k}_{0}$ be a linear extension of $\mathfrak{g}_{0}$ of dimension $2 n$ with basis $\left\{z_{1}, \ldots, z_{n}, w_{1}, \ldots, w_{n}\right\}$. Then we define a Lie superalgebra structure $\mathfrak{g}=\mathfrak{g}_{0} \oplus \mathfrak{g}_{1}$ of dimension $2 n$ and a Lie superalgebra structure $\mathfrak{k}=\mathfrak{k}_{0} \oplus \mathfrak{g}_{1}$ of dimension $3 n$ by setting for the values of the super-bracket on these generators: 
$\left\{\begin{array}{l}\left\{y_{i}, y_{i}\right\}=z_{i},\left[w_{i}, y_{i}\right]=y_{i},\left[w_{i}, z_{i}\right]=2 z_{i} \text { for any } 1 \leq i \leq n, \\ \text { the brackets are zero for all other pairs of generators. }\end{array}\right.$

By construction $\mathfrak{g}_{0}$ is a subsuperalgebra of $\mathfrak{k}_{0}$ and $\mathfrak{g}=\mathfrak{g}_{0} \oplus \mathfrak{g}_{1}$ is a subsuperalgebra of $\mathfrak{k}$. It is clear by PBW theorem (see Theorem 6.1.2 in [14]) that the enveloping algebra $\mathcal{U}(\mathfrak{k})$ is isomorphic to $\widetilde{U}_{n}(\mathbb{C})$ of GK-dim $2 n$. Moreover the subalgebra $\mathcal{U}\left(\mathfrak{k}_{0}\right)$ is isomorphic to $U_{n}(\mathbb{C})$ of $\mathrm{GK}$-dim $2 n$, and the subalgebra $\mathcal{U}(\mathfrak{g})$ is isomorphic to $\widetilde{\mathcal{O}}\left(\mathbb{C}^{n}\right)$ of GK-dim $n$.

(ii) We have for the usual Weyl algebra the classical isomorphism $A_{n}(\mathbb{C}) \simeq$ $A_{1}(\mathbb{C})^{\otimes n}$. We also have $\widetilde{A}_{n}(\mathbb{C}) \simeq \widetilde{A}_{1}(\mathbb{C})^{\otimes}$ in the tensor category of superalgebras assigning the parity 1 to the generators $x_{i}$ and $y_{i}$.

(iii) All isomorphims and tensor products considered in the following are in the tensor category of associative algebras.

1.2.4. Remark. All algebras introduced in 1.2.1 can be described as iterated skew polynomial algebras over $\mathbb{C}$; then they are noetherian domains, and therefore they admit a skewfield of fractions. The skewfield Frac $A_{n}(\mathbb{C})=$ Frac $U_{n}(\mathbb{C})$ is the well known Weyl skewfield $D_{n}(\mathbb{C})$, with center $\mathbb{C}$. Similarly we deduce from $(4)$ that $\operatorname{Frac} \widetilde{A}_{n}(\mathbb{C})=$ Frac $\widetilde{U}_{n}(\mathbb{C})$, and it is proved in Proposition 3.3.1 of [18] that its center is also $\mathbb{C}$. In parallel with Proposition 1.1.3. we have for these skewfields the following canonical form.

1.2.5. Theorem. For any integer $n \geq 1$, we have the following isomorphisms:

(i) If $n=2 p$ is even, then Frac $\widetilde{A}_{n}(\mathbb{C})=\operatorname{Frac}\left(\widetilde{A}_{2}(\mathbb{C})^{\otimes p}\right)$.

(ii) If $n=2 p+1$ is odd, then Frac $\widetilde{A}_{n}(\mathbb{C})=\operatorname{Frac}\left(\widetilde{A}_{2}(\mathbb{C})^{\otimes p} \otimes A_{1}(\mathbb{C})\right)$.

Proof. We can suppose $n \geq 1$. We consider the subalgebra $\widetilde{U}_{n}(\mathbb{C})$ defined in 1.2.1 with generators $w_{1}, \ldots, w_{n}, y_{1}, \ldots y_{n}$ and relations (4). As in the proof of Proposition 1.1.3, we introduce: $y_{1}^{\prime}=y_{1}, y_{2}^{\prime}=y_{2}$ and $y_{i}^{\prime}=y_{1} y_{2} y_{i}$ for $3 \leq$ $i \leq n$. Then we define: $w_{1}^{\prime}=w_{1}-\left(w_{3}+\cdots+w_{n}\right), w_{2}^{\prime}=w_{2}-\left(w_{3}+\cdots+w_{n}\right)$ and $w_{i}^{\prime}=w_{i}$ for $3 \leq i \leq n$. Obvious calculations give:

$$
\begin{aligned}
& \left\{\begin{array}{l}
y_{1}^{\prime} y_{2}^{\prime}=-y_{2}^{\prime} y_{1}^{\prime}, \quad w_{1}^{\prime} w_{2}^{\prime}=w_{2}^{\prime} w_{1}^{\prime}, \quad y_{1}^{\prime} w_{2}^{\prime}=w_{2}^{\prime} y_{1}^{\prime}, \quad y_{2}^{\prime} w_{1}^{\prime}=w_{1}^{\prime} y_{2}^{\prime}, \\
{\left[w_{1}^{\prime}, y_{1}^{\prime}\right]=y_{1}^{\prime}, \quad\left[w_{2}^{\prime}, y_{2}^{\prime}\right]=y_{2}^{\prime} .}
\end{array}\right. \\
& \left\{\begin{array}{c}
y_{1}^{\prime} y_{j}^{\prime}=y_{j}^{\prime} y_{1}^{\prime}, \quad y_{2}^{\prime} y_{j}^{\prime}=y_{j}^{\prime} y_{2}^{\prime}, \quad y_{1}^{\prime} w_{j}^{\prime}=w_{j}^{\prime} y_{1}^{\prime}, \quad \\
y_{2}^{\prime} w_{j}^{\prime}=w_{j}^{\prime} y_{2}^{\prime}, \quad w_{1}^{\prime} w_{j}^{\prime}=w_{j}^{\prime} w_{1}^{\prime}, \quad w_{2}^{\prime} w_{j}^{\prime}=w_{j}^{\prime} w_{2}^{\prime}, \quad \text { for } 3 \leq j \leq n .
\end{array}\right.
\end{aligned}
$$

We calculate for $\ell=1$ or 2 and any $3 \leq j \leq n$ the commutator: $\left[w_{\ell}^{\prime}, y_{j}^{\prime}\right]=$ $\left[w_{\ell}, y_{1} y_{2} y_{j}\right]-\sum_{i=3}^{n}\left[w_{i}, y_{1} y_{2} y_{j}\right]=y_{1} y_{2} y_{j}-\left[w_{j}, y_{1} y_{2} y_{j}\right]=0$ to conclude $:$

$$
y_{j}^{\prime} w_{1}^{\prime}=w_{1}^{\prime} y_{j}^{\prime} \text { and } y_{j}^{\prime} w_{2}^{\prime}=w_{2}^{\prime} y_{j}^{\prime} \text { for any } 3 \leq j \leq n .
$$

Moreover:

$$
\begin{aligned}
& {\left[w_{j}^{\prime}, y_{j}^{\prime}\right]=y_{j}^{\prime} } \text { for any } 3 \leq j \leq n, \\
& y_{i}^{\prime} y_{j}^{\prime}=-y_{j}^{\prime} y_{i}^{\prime}, \quad w_{i}^{\prime} w_{j}^{\prime}=w_{j}^{\prime} w_{i}^{\prime}, \quad w_{i}^{\prime} y_{j}^{\prime}=y_{j}^{\prime} w_{i}^{\prime}, \quad \text { for } 3 \leq i \neq j \leq n .
\end{aligned}
$$

We denote by $V$ the subalgebra of $\widetilde{U}_{n}(\mathbb{C})$ generated by $y_{1}^{\prime}, \ldots, y_{n}^{\prime}, w_{1}^{\prime}, \ldots, w_{n}^{\prime}$, by $W$ the subalgebra of $V$ generated by $y_{1}^{\prime},, y_{2}^{\prime}, w_{1}^{\prime}, w_{2}^{\prime}$, and by $W^{\prime}$ the 
subalgebra of $V$ generated by $y_{3}^{\prime}, \ldots, y_{n}^{\prime}, w_{3}^{\prime}, \ldots, w_{n}^{\prime}$. It follows from (5) that $W^{\prime} \simeq \widetilde{U}_{2}(\mathbb{C})$, from $(8)$ and $(9)$ that $W^{\prime \prime} \simeq \widetilde{U}_{n-2}(\mathbb{C})$, and from (7) and (9) that $V \simeq W \otimes W^{\prime}$. Since it is clear that Frac $V=\operatorname{Frac} \widetilde{U}_{n}(\mathbb{C})$, we deduce that $\operatorname{Frac} \widetilde{U}_{n}(\mathbb{C}) \simeq \operatorname{Frac}\left(\widetilde{U}_{2}(\mathbb{C}) \otimes \widetilde{U}_{n-2}(\mathbb{C})\right)$. We conclude that Frac $\widetilde{A}_{n}(\mathbb{C}) \simeq \operatorname{Frac}\left(\widetilde{A}_{2}(\mathbb{C}) \otimes \widetilde{A}_{n-2}(\mathbb{C})\right)$ and finish by induction on $n$.

\section{The Lie superalgeBra orp $(1,2 n)$ And its EnVeloping Algebra}

2.1. Generators and relations. We refer for instance to [6], [15], 2]. The basefield is $\mathbb{C}$. For any $n \geq 1$, the odd part $\mathfrak{g}_{\overline{1}}$ of $\mathfrak{g}=\mathfrak{o} \mathfrak{s p}(1,2 n)$ is a vector space of dimension $2 n$ and the even part $\mathfrak{g}_{0}$ is isomorphic to the symplectic Lie algebra $\mathfrak{s p}(2 n)$ of dimension $2 n^{2}+n$. As a Lie superalgebra, osp $(1,2 n)$ is generated by the elements $b_{i}^{ \pm}(1 \leq i \leq n)$ of a basis of $\mathfrak{g}_{\overline{1}}$. Then the elements $\left\{b_{j}^{ \pm}, b_{k}^{ \pm}\right\}(1 \leq j \leq k \leq n)$ and $\left\{b_{j}^{+}, b_{k}^{-}\right\}(1 \leq j, k \leq n)$ form a basis of $\mathfrak{g}_{\overline{0}}$. The brackets in $\mathfrak{o s p}(1,2 n)$ are given by the "para-Bose" relations on the generators:

$$
\begin{gathered}
{\left[\left\{b_{j}^{\xi}, b_{k}^{\eta}\right\}, b_{\ell}^{\epsilon}\right]=(\epsilon-\xi) \delta_{j \ell} b_{k}^{\eta}+(\epsilon-\eta) \delta_{k \ell} b_{j}^{\xi}} \\
{\left[\left\{b_{i}^{\xi}, b_{j}^{\eta}\right\},\left\{b_{k}^{\epsilon}, b_{\ell}^{\varphi}\right\}\right]=(\epsilon-\eta)} \\
\delta_{j k}\left\{b_{i}^{\xi}, b_{\ell}^{\varphi}\right\}+(\epsilon-\xi) \delta_{i k}\left\{b_{j}^{\eta}, b_{\ell}^{\varphi}\right\} \\
+(\varphi-\eta) \delta_{j \ell}\left\{b_{i}^{\xi}, b_{k}^{\epsilon}\right\}+(\varphi-\xi) \delta_{i \ell}\left\{b_{j}^{\eta}, b_{k}^{\epsilon}\right\},
\end{gathered}
$$

with $1 \leq i, j, k, \ell \leq n$ and $\epsilon, \varphi, \xi, \eta$ are \pm . By PBW theorem, the enveloping algebra $\mathcal{U}(\mathfrak{o s p}(1,2 n))$ is generated by the $2 n^{2}+n$ elements:

$$
\begin{aligned}
& b_{i}^{ \pm} \text {and } k_{i}:=\frac{1}{2}\left\{b_{i}^{-}, b_{i}^{+}\right\} \text {for } 1 \leq i \leq n, \\
& a_{i j}^{ \pm}:=\frac{1}{2}\left\{b_{i}^{ \pm}, b_{j}^{ \pm}\right\} \text {for } 1 \leq i<j \leq n, \\
& s_{i j}:=\frac{1}{2}\left\{b_{i}^{-}, b_{j}^{+}\right\} \text {and } t_{i j}:=\frac{1}{2}\left\{b_{i}^{+}, b_{j}^{-}\right\} \text {for } 1 \leq i<j \leq n,
\end{aligned}
$$

of $\mathfrak{o s p}(1,2 n)$. In particular, denoting

$$
c_{i}^{ \pm}:=\frac{1}{2}\left\{b_{i}^{ \pm}, b_{i}^{ \pm}\right\} \text {for } 1 \leq i \leq n,
$$

the enveloping algebra $\mathcal{U}(\mathfrak{s p}(2 n))$ of the even part $\mathfrak{g}_{\overline{0}}$ is the subalgebra of $\mathcal{U}(\mathfrak{o s p}(1,2 n))$ generated by the $2 n^{2}+n$ elements $\left(b_{i}^{ \pm}\right)^{2}=c_{i}^{ \pm}, k_{i}$ for $1 \leq$ $i \leq n$, and $a_{i j}^{ \pm}, s_{i j}, t_{i j}$ for $1 \leq i<j \leq n$. The commutation relations in the associative algebra $\mathcal{U}(\mathfrak{o s p}(1,2 n))$ are deduced from (10) and (11) taking $\{x, y\}=x y+y x$ if $x, y \in \mathfrak{g}_{\overline{1}}$, and $[x, y]=x y-y x$ otherwise. In particular commutation relations between the generators defined by $(12),(13),(14)$, (15) are obtained by injecting them in relations (10) and (11). For instance:

$$
\begin{array}{lr}
b_{i}^{+} b_{j}^{+}+b_{j}^{+} b_{i}^{+}=2 a_{i j}^{+} & \text {for all } 1 \leq i<j \leq n, \\
{\left[c_{i}^{+}, b_{j}^{+}\right]=\left[c_{i}^{+}, c_{j}^{+}\right]=0} & \text { for all } 1 \leq i<j \leq n, \\
{\left[a_{i j}^{+}, b_{\ell}^{+}\right]=\left[a_{i j}^{+}, c_{\ell}^{+}\right]=0} & \text { for all } 1 \leq i<j \leq n, 1 \leq \ell \leq n, \\
{\left[a_{i j}^{+}, a_{k \ell}^{+}\right]=0} & \text { for all } 1 \leq i<j \leq n, 1 \leq k<\ell \leq n .
\end{array}
$$


Similarly with (10) and (14), we deduce for all $1 \leq i<j \leq n$ and $1 \leq k \leq n$ :

$$
\left[t_{i j}, b_{j}^{+}\right]=b_{i}^{+} \text {and }\left[t_{i j}, b_{k}^{+}\right]=0 \text { if } k \neq j .
$$

The relations involving the $t_{i j}$ 's are obtained from (11), 13) and (14), which give for $1 \leq i<j \leq n$ and $1 \leq k<\ell \leq n$ :

$$
\begin{aligned}
& {\left[t_{i j}, a_{k \ell}^{+}\right]=\delta_{j k} \frac{1}{2}\left\{b_{i}^{+}, b_{\ell}^{+}\right\}+\delta_{j \ell} \frac{1}{2}\left\{b_{i}^{+}, b_{k}^{+}\right\},} \\
& {\left[t_{i j}, t_{k \ell}\right]=\delta_{j k} \frac{1}{2}\left\{b_{i}^{+}, b_{\ell}^{-}\right\}-\delta_{i \ell} \frac{1}{2}\left\{b_{k}^{+}, b_{j}^{-}\right\},}
\end{aligned}
$$

or in other words:

$$
\begin{aligned}
{\left[t_{i j}, a_{k \ell}^{+}\right]=0 \text { if } j \neq k, j \neq \ell } & (1 \leq i<j \leq n, 1 \leq k<\ell \leq n), \\
{\left[t_{i j}, a_{j \ell}^{+}\right]=a_{i \ell}^{+} } & (1 \leq i<j<\ell \leq n), \\
{\left[t_{i j}, a_{i j}^{+}\right]=c_{i}^{+} } & (1 \leq i<j \leq n), \\
{\left[t_{i j}, a_{k j}^{+}\right]=a_{i k}^{+} \text {if } i<k } & (1 \leq i<j \leq n, 1 \leq k<j \leq n,), \\
{\left[t_{i j}, a_{k j}^{+}\right]=a_{k i}^{+} \text {if } k<i } & (1 \leq i<j \leq n, 1 \leq k<j \leq n,) . \\
{\left[t_{i j}, t_{j \ell}=t_{i \ell}\right.} & (1 \leq i<j<\ell \leq n), \\
{\left[t_{i j}, t_{k i}\right]=-t_{k j} } & (1 \leq k<i<j \leq n), \\
{\left[t_{i j}, t_{k \ell}\right]=0 \text { if } j \neq k, i \neq \ell } & (1 \leq i<j \leq n, 1 \leq k<\ell \leq n) .
\end{aligned}
$$

The action of the generators $k_{i}$ follows from 10 and 12 :

$$
\begin{array}{lrl}
{\left[k_{i}, k_{j}\right]=0} & (1 \leq i, j \leq n), \\
{\left[k_{i}, b_{i}^{+}\right]=b_{i}^{+},\left[k_{i}, b_{j}^{ \pm}\right]=0} & (1 \leq i \neq j \leq n),
\end{array}
$$

then with (13) and (14), for all $1 \leq i<j \leq n$ and $1 \leq \ell \leq n$ :

$$
\left\{\begin{array} { l } 
{ [ k _ { i } , a _ { i j } ^ { + } ] = a _ { i j } ^ { + } , } \\
{ [ k _ { j } , a _ { i j } ^ { + } ] = a _ { i j } ^ { + } , } \\
{ [ k _ { \ell } , a _ { i j } ^ { + } ] = 0 \text { if } \ell \neq i , j . }
\end{array} \quad \left\{\begin{array}{l}
{\left[k_{i}, t_{i j}\right]=t_{i j},} \\
{\left[k_{j}, t_{i j}\right]=-t_{i j},} \\
{\left[k_{\ell}, t_{i j}\right]=0 \text { if } \ell \neq i, j .}
\end{array}\right.\right.
$$

2.2. Chevalley generators and Serre relations. Following [15] (up to a renormalization of the $n$-th generator by $\sqrt{2}$ ), we introduce:

$$
t_{i}:=t_{i, i+1} \text { for any } 1 \leq i \leq n-1, \text { and } t_{n}:=b_{n}^{+},
$$

which satisfy:

$$
\begin{array}{lr}
{\left[t_{i}, t_{j}\right]=0 \text { if }|i-j|>1} & (1 \leq i, j \leq n), \\
{\left[t_{i}, t_{i+1}\right]=t_{i, i+2}} & (1 \leq i \leq n-2), \\
{\left[t_{n-1}, t_{n}\right]=b_{n-1}^{+} .} &
\end{array}
$$

It also follows from that for any $1 \leq i \leq n-1$ and $1 \leq j \leq n$ :

$$
\left[t_{i}, b_{i+1}^{+}\right]=b_{i}^{+} \text {and }\left[t_{i}, b_{j}^{+}\right]=0 \text { if } j \neq i+1 .
$$


By straightforward calculations using (29), (35), (36), (37), the elements $t_{1}, \ldots, t_{n}$ defined in (34) satisfy the following Serre identities which already appear as (2.11) in [15]:

$$
\begin{array}{ll}
t_{i}^{2} t_{i+1}-2 t_{i} t_{i+1} t_{i}+t_{i+1} t_{i}^{2}=0 & (1 \leq i \leq n-1), \\
t_{i}^{2} t_{i-1}-2 t_{i} t_{i-1} t_{i}+t_{i-1} t_{i}^{2}=0 & (2 \leq i \leq n-1), \\
t_{n}^{3} t_{n-1}-\left(t_{n}^{2} t_{n-1} t_{n}+t_{n} t_{n-1} t_{n}^{2}\right)+t_{n-1} t_{n}^{3}=0 . &
\end{array}
$$

\subsection{Enveloping algebra of the nilpotent subsuperalgebra $\mathfrak{n}^{+}$.}

2.3.1. Definition of $\mathfrak{n}^{+}$. We define $\mathfrak{n}^{+}:=\mathfrak{o s p}^{+}(1,2 n)$ as the subsuperalgebra of $\mathfrak{o s p}(1,2 n)$ generated by Chevalley generators $t_{1}, t_{2}, \ldots, t_{n}$. By (34), (37) and (38), $\mathfrak{n}^{+}$contains the elements $b_{1}^{+}, b_{2}^{+}, \ldots, b_{n}^{+}$. Hence by (13), $\mathfrak{n}^{+}$ contains the elements $a_{i j}^{+}$for $1 \leq i<j \leq n$. It also follows inductively from (28) and (36) that $\mathfrak{n}^{+}$contains the elements $t_{i j}$ for $1 \leq i<j \leq n$. Hence:

$$
\mathfrak{n}^{+}=\mathfrak{n}_{\overline{0}}^{+} \oplus \mathfrak{n}_{\overline{1}}^{+}
$$

where $\mathfrak{n}_{\overline{1}}^{+}$is the $\mathbb{C}$-vector space with basis $\left(b_{i}^{+}\right)_{1 \leq i \leq n}$, and $\mathfrak{n}_{\overline{0}}^{+} \simeq \mathfrak{s p}^{+}(2 n)$ is the Lie subalgebra of $\mathfrak{g}_{\overline{0}} \simeq \mathfrak{s p}(2 n)$ with basis $\left(c_{i}^{+}, a_{i j}^{+}, t_{i j}\right)_{1 \leq i<j \leq n}$. Then:

$$
\operatorname{dim} \mathfrak{n}_{\overline{0}}^{+}=\operatorname{dim} \mathfrak{s p}^{+}(2 n)=n^{2} \quad \text { and } \quad \operatorname{dim} \mathfrak{n}_{1}^{+}=n .
$$

Let us observe that relations (17), (18) and (19) imply that the Lie subalgebra with basis $\left(c_{i}^{+}, a_{i j}^{+}\right)_{1 \leq i<j \leq n}$ in $\mathfrak{n}_{\overline{0}}^{+}$is abelian of dimension $n(n+1) / 2$.

2.3.2. Description of $\mathcal{U}\left(\mathfrak{n}^{+}\right)$as an iterated skew polynomial algebra. We denote by $U^{+}$the enveloping algebra $\mathcal{U}\left(\mathfrak{n}^{+}\right)$of $\mathfrak{n}^{+}$. By PBW theorem $U^{+}$is generated as an associative algebra over $\mathbb{C}$ by the $n^{2}$ elements $\mathrm{b}_{i}^{+}, a_{i j}^{+}$and $t_{i j}$ for $1 \leq i<j \leq n$. The relations detailed in 2.1 allow us to describe $U^{+}$as an iterated skew polynomial extension. We start with $A:=\mathbb{C}\left[a_{i j}^{+}\right]_{1 \leq i<j \leq n}$, which is by $(19)$ a commutative polynomial algebra in $n(n-1) / 2$ variables. Then we set:

$$
B_{0}:=A, \quad B_{1}:=A\left[b_{1}^{+}\right], \quad B_{j}:=B_{j-1}\left[b_{j}^{+} ; \sigma_{j}, \delta_{j}\right] \text { for } 2 \leq j \leq n,
$$

where $\sigma_{j}$ is the automorphism of $B_{j-1}$ and $\delta_{j}$ is the $\sigma_{j}$-derivation of $B_{j-1}$ defined by $\sigma_{j}\left(a_{k \ell}^{+}\right)=a_{k \ell}^{+}$and $\delta_{j}\left(a_{k \ell}^{+}\right)=0$ for all $1 \leq k<\ell \leq n$, and $\sigma_{j}\left(b_{i}^{+}\right)=-b_{i}^{+}$and $\delta_{j}\left(b_{i}^{+}\right)=2 a_{i j}^{+}$for any $1 \leq i<j$, in order to translate relations (16) and (18). At the last step we denote:

$$
B:=B_{n}=\mathbb{C}\left[a_{i j}^{+}\right]\left[b_{1}^{+}\right]\left[b_{2}^{+} ; \sigma_{2}, \delta_{2}\right] \cdots\left[b_{n}^{+} ; \sigma_{n}, \delta_{n}\right] .
$$

Then we add the generators $t_{i j}$ for $1 \leq i<j \leq n$ inductively:

$$
\begin{aligned}
& R_{1}:=B\left[t_{1,2} ; d_{1,2}\right]\left[t_{1,3} ; d_{1,3}\right] \cdots\left[t_{1, n} ; d_{1, n}\right] \\
& R_{i}:=R_{i-1}\left[t_{i, i+1} ; d_{i, i+1}\right]\left[t_{i, i+2} ; d_{i, i+2}\right] \cdots\left[t_{i, n} ; d_{i, n}\right], \quad(2 \leq i \leq n-2) \\
& R_{n-1}:=R_{n-2}\left[t_{n-1, n} ; d_{n-1, n}\right]=U^{+}
\end{aligned}
$$


where the derivations $d_{i j}$ are suitably defined to account for relations 20 and (23) to 30). To sum up we conclude:

$$
\begin{aligned}
& A=\mathbb{C}\left[a_{i j}^{+}\right]_{1 \leq i<j<n}, \\
& B=A\left[b_{i}^{+} ; \sigma_{i}, \delta_{i}\right]_{1 \leq i \leq n}, \\
& U^{+}=B\left[t_{i j} ; d_{i j}\right]_{1 \leq i<j \leq n} .
\end{aligned}
$$

Let us recall that in this presentation, $A$ is commutative and the $b_{i}^{+}$'s commute with the elements of $A$.

2.3.3. Restriction to the even part. Because of relations (17) and (18), we can consider in $B$ the commutative subalgebra $C$ generated over $A$ by the elements $c_{k}^{+}=\left(b_{k}^{+}\right)^{2}$ for $1 \leq k \leq n$. It follows from previous results that:

$$
\begin{aligned}
& C=\mathbb{C}\left[a_{i j}^{+}, c_{k}^{+}\right]_{1 \leq i<j<n, 1 \leq k \leq n}, \\
& \mathcal{U}\left(\mathfrak{n}_{\overline{0}}^{+}\right)=C\left[t_{i j} ; d_{i j}\right]_{1 \leq i<j \leq n} .
\end{aligned}
$$

\subsection{Enveloping algebra of the solvable Borel subsuperalgebra $\mathfrak{b}^{+}$.}

2.4.1. Definition of $\mathfrak{b}^{+}$. The Cartan Lie subalgebra $\mathfrak{h}$ of $\mathfrak{g}_{0}$ is the Lie subalgebra with basis $\left(k_{i}\right)_{1 \leq i \leq n}$, which is abelian by relation (31). The positive Borel subsuperalgebra $\mathfrak{b}^{+}$is defined as the subsuperalgebra of $\mathfrak{o s p}(1,2 n)$ generated by $\mathfrak{n}^{+}$and $\mathfrak{h}$. We have clearly:

$$
\mathfrak{b}^{+}=\mathfrak{n}^{+} \oplus \mathfrak{h}=\mathfrak{b}_{\overline{0}}^{+} \oplus \mathfrak{n}_{\overline{1}}^{+}, \quad \text { with } \mathfrak{b}_{\overline{0}}^{+}:=\mathfrak{n}_{\overline{0}}^{+} \oplus \mathfrak{h},
$$

where the even part $\mathfrak{b}_{\overline{0}}^{+}$is the positive Borel Lie subalgebra of $\mathfrak{g}_{\overline{0}} \simeq \mathfrak{s p}(2 n)$.

2.4.2. Description of $\mathcal{U}\left(\mathfrak{b}^{+}\right)$as an iterated polynomial algebra. We denote by $U_{b}^{+}$the enveloping algebra $\mathcal{U}\left(\mathfrak{b}^{+}\right)$of $\mathfrak{b}^{+}$. The commutation relations of the $k_{i}$ 's with the generators of $U^{+}$given by formulas (31) to (33) allow an easy description of $U_{b}^{+}$as the following iterated skew polynomial algebra:

$$
U_{b}^{+}=U^{+}\left[k_{1}, \Delta_{1}\right]\left[k_{2}, \Delta_{2}\right] \cdots\left[k_{n}, \Delta_{n}\right],
$$

where the derivations $\Delta_{i}$ vanish on all $k_{j}$ 's and satisfy $\Delta_{i}\left(b_{i}^{+}\right)=b_{i}^{+}$and $\Delta_{i}\left(b_{j}^{+}\right)=0$ for any $1 \leq j \neq i \leq n, \Delta_{i}\left(a_{i j}^{+}\right)=a_{i j}^{+}$and $\Delta_{i}\left(t_{i j}\right)=t_{i j}$ for $1 \leq i<j \leq n, \Delta_{i}\left(a_{j i}^{+}\right)=a_{j i}^{+}$and $\Delta_{i}\left(t_{j i}\right)=-t_{j i}$ for $1 \leq j<i \leq n$, $\Delta_{i}\left(a_{k \ell}^{+}\right)=0$ and $\Delta_{i}\left(t_{k \ell}\right)=0$ for $k \neq i, \ell \neq i$.

2.4.3. Restriction to the even part. It follows from previous results that, with notations (49) and (48):

$$
\mathcal{U}\left(\mathfrak{b}_{\overline{0}}^{+}\right)=\mathcal{U}\left(\mathfrak{n}_{\overline{0}}^{+}\right)\left[k_{i} ; \Delta_{i}\right]_{1 \leq i \leq n}=C\left[t_{i j} ; d_{i j}\right]_{1 \leq i<j \leq n}\left[k_{i} ; \Delta_{i}\right]_{1 \leq i \leq n} .
$$

\section{Proof of the MAIN THEOREM}

3.1. Some general technical results. We will use the following easy lemmas. 
3.1.1. Lemma. Let $A$ be a commutative $\mathbb{C}$-algebra, a an element of $A$, and $R$ the iterated skew polynomial algebra generated over $A$ by two generators $x_{1}$ and $x_{2}$ commuting with the elements of $A$ and with relation $x_{1} x_{2}+x_{2} x_{1}=$ $2 a$. Then the element $y=x_{1} x_{2}-a$ satisfies $x_{1} y=-y x_{1}$ and $x_{2} y=-y x_{2}$.

Proof. Obvious calculation.

3.1.2. Lemma. Let $A$ be a commutative $\mathbb{C}$-algebra. Let $\left(a_{i j}\right)_{1 \leq i<j \leq n}$ be a family of elements of $A$, and $R$ the iterated skew polynomial algebra generated over $A$ by $n$ generators $x_{1}, x_{2}, \ldots, x_{n}$ commuting with the elements of $A$ and with relations $x_{i} x_{j}+x_{j} x_{i}=2 a_{i j}$ for all $1 \leq i<j \leq n$. Then the $n-1$ elements $y_{i}=x_{1} x_{i}-a_{1 i}$ for any $2 \leq i \leq n$ satisfy:

$$
x_{1} y_{i}=-y_{i} x_{1} \quad \text { and } \quad y_{i} y_{j}+y_{j} y_{i}=2\left(a_{1 i} a_{1 j}-a_{i j} x_{1}^{2}\right) .
$$

Proof. The first identity follows from previous lemma. For the second one, we observe firstly that:

$$
\begin{aligned}
x_{j} y_{i} & =x_{j} x_{1} x_{i}-x_{j} a_{1 i}=\left(-x_{1} x_{j}+2 a_{1 j}\right) x_{i}-a_{1 i} x_{j} \\
& =-x_{1} x_{j} x_{i}+2 a_{1 j} x_{i}-a_{1 i} x_{j}=x_{1} x_{i} x_{j}-2 a_{i j} x_{1}+2 a_{1 j} x_{i}-a_{1 i} x_{j} \\
& =\left(x_{1} x_{i}-a_{1 i}\right) x_{j}-2 a_{i j} x_{1}+2 a_{1 j} x_{i}=y_{i} x_{j}-2 a_{i j} x_{1}+2 a_{1 j} x_{i} .
\end{aligned}
$$

We use this identity and Lemma 3.1.1 to calculate:

$$
\begin{aligned}
y_{j} y_{i} & =\left(x_{1} x_{j}-a_{1 j}\right) y_{i}=x_{1}\left(y_{i} x_{j}-2 a_{i j} x_{1}+2 a_{1 j} x_{i}\right)-a_{1 j} y_{i} \\
& =-y_{i} x_{1} x_{j}-2 a_{i j} x_{1}^{2}+2 a_{1 j} x_{1} x_{i}-a_{1 j} y_{i} \\
& =-y_{i}\left(x_{1} x_{j}-a_{1 j}\right)-2 a_{1 j} y_{i}-2 a_{i j} x_{1}^{2}+2 a_{1 j} x_{1} x_{i} \\
& =-y_{i} y_{j}-2 a_{1 j} y_{i}-2 a_{i j} x_{1}^{2}+2 a_{1 j} x_{1} x_{i} \\
& =-y_{i} y_{j}+2 a_{1 j}\left(x_{1} x_{i}-y_{i}\right)-2 a_{i j} x_{1}^{2} .
\end{aligned}
$$

We conclude that $y_{j} y_{i}=-y_{i} y_{j}+2 a_{1 j} a_{1 i}-2 a_{i j} x_{1}^{2}$.

\subsection{Enveloping skewfield of the Lie subsuperalgebra $\mathfrak{n}^{+}$.}

3.2.1. Notations. Recalling the description (44), (45), (46) of $U^{+}$, we introduce the skewfields of rational functions:

$$
\begin{aligned}
K & :=\operatorname{Frac} A=\mathbb{C}\left(a_{i j}^{+}\right)_{1 \leq i<j \leq n}, \\
L & :=\operatorname{Frac} B=K\left(b_{i}^{+} ; \sigma_{i}, \delta_{i}\right)_{1 \leq i \leq n}, \\
F & :=\operatorname{Frac} U^{+}=L\left(t_{i j} ; d_{i j}\right)_{1 \leq i<j \leq n} .
\end{aligned}
$$

3.2.2. Notations. We define the following families of elements of $B$ :

$$
y_{1}:=b_{1}^{+}, \quad x_{1, i}:=b_{i}^{+}, \quad u_{1, i, j}:=a_{i, j}^{+} \quad \text { for all } 1 \leq i<j \leq n,
$$

and more generally for any $1 \leq k \leq n$ :

$$
\begin{cases}u_{k, i, j}:=u_{k-1, k-1, i} u_{k-1, k-1, j}-u_{k-1, i, j} y_{k-1}^{2} & \text { for all } k \leq i<j \leq n \\ x_{k, i}:=x_{k-1, k-1} x_{k-1, i}-u_{k-1, k-1, i} & \text { for any } k \leq i \leq n \\ y_{k}:=x_{k, k} & \end{cases}
$$


It is clear that we have with notation (42):

$$
y_{k} \in B_{k}, x_{k, i} \in B_{i}, u_{k, i, j} \in B_{k-1} \quad \text { for all } 1 \leq k \leq n, k \leq i<j \leq n .
$$

\subsubsection{Lemma.}

(i) The elements $y_{1}, y_{2}, \ldots, y_{n}$ of $B$ satisfy the relations:

$$
\begin{cases}y_{k-1} y_{k}=-y_{k} y_{k-1} & \text { for any } 2 \leq k \leq n, \\ y_{k} y_{\ell}=y_{\ell} y_{k} & \text { for all } 1 \leq k, \ell \leq n, \ell \neq k \pm 1, \\ y_{k} a_{i j}^{+}=a_{i j}^{+} y_{k} & \text { for all } 1 \leq k \leq n, 1 \leq i<j \leq n .\end{cases}
$$

(ii) For any $1 \leq k \leq n$, the elements $u_{k, i, j}$ with $k \leq i<j \leq n$ are in the commutative subalgebra $A\left[y_{1}^{2}, y_{2}^{2}, \ldots, y_{k-1}^{2}\right]$ of $B$,

Proof. By iterative application of Lemma 3.1.2, we have for any $1 \leq k \leq n$ :

$$
\begin{array}{lr}
x_{k-1, k-1} x_{k, i}=-x_{k, i} x_{k-1, k-1} & \text { for any } 2 \leq k \leq i \leq n, \\
x_{k, i} x_{k, j}+x_{k, j} x_{k, i}=2 u_{k, i, j} & \text { for all } k \leq i<j \leq n .
\end{array}
$$

We deduce by induction on $\ell$ that, for any $1 \leq \ell \leq n$ :

$$
\begin{cases}y_{\ell} x_{\ell+1, i}=-x_{\ell+1, i} y_{\ell} & \text { for any } \ell+1 \leq i \leq n, \\ y_{\ell} x_{k, i}=x_{k, i} y_{\ell} & \text { for any } \ell+2 \leq k \leq i \leq n, \\ y_{\ell} u_{k, i, j}=u_{k, i, j} y_{\ell} & \text { for any } 1 \leq k \leq i<j \leq n .\end{cases}
$$

Point (i) of the lemma follows obviously from these relations and point (ii) follows by iterative definition (55) of the $u_{k, i, j}$ 's.

\subsubsection{Proposition.}

(i) The subalgebra of $B$ generated over $\mathbb{C}$ by $y_{1}, \ldots, y_{n}$ is isomophic to $\mathcal{O}_{\Lambda}\left(\mathbb{C}^{n}\right)$, where all entries of the symmetric matrix $\Lambda=\left(\lambda_{i j}\right)_{1 \leq i, j \leq n}$ are equal to 1 except $\lambda_{i, i+1}=-1=\lambda_{i+1, i}$ for any $1 \leq i \leq n-1$.

(ii) Its skewfield of fractions $Q$ satisfies:

$$
\begin{aligned}
& L=Q\left(a_{i j}^{+}\right)_{1 \leq i<j \leq n}, \\
& Q \simeq \operatorname{Frac}\left(\widetilde{\mathcal{O}}\left(\mathbb{C}^{n}\right)\right),
\end{aligned}
$$

where each $a_{i j}^{+}$commutes with the elements of $Q$.

Proof. By induction from relations (55), we prove that for any $1 \leq k \leq n$,

$$
y_{k}=\left(y_{k-1} y_{k-2} \cdots y_{2} y_{1}\right) b_{k}^{+}+r_{k-1},
$$

where the term $r_{k-1}$ lies in the subalgebra of $B_{k-1}$ generated by $y_{1}, \ldots, y_{k-1}$ and the $a_{i j}^{+}$'s for $1 \leq i<j \leq k$. It follows that the skewfield $L$ defined by (52) is also the skewfield of rational functions generated over $K$ by $y_{1}, \ldots, y_{n}$. Then the commutation relations of Lemma 3.2 .3 imply point (i) and assertion (60) of point (ii). In order to prove assertion (61), we suppose that $n \geq 3$ and define: $y_{1}^{\prime}=y_{1}, y_{2}^{\prime}=y_{2}, y_{3}^{\prime}=y_{1} y_{3}$ and $y_{i}^{\prime}=y_{i}$ for any $4 \leq i \leq n$. It is clear that the subfield of $Q$ generated by the elements $\left(y_{i}^{\prime}\right)_{1 \leq i \leq n}$ is equal to $Q$. Moreover by straightforward calculations, we have: 


$$
\begin{cases}y_{1}^{\prime} y_{2}^{\prime}=-y_{2}^{\prime} y_{1}^{\prime}, \quad y_{1}^{\prime} y_{i}^{\prime}=y_{i}^{\prime} y_{1}^{\prime}, \quad y_{2}^{\prime} y_{i}^{\prime}=y_{2}^{\prime} y_{1}^{\prime}, & \text { for any } 3 \leq i \leq n, \\ y_{3}^{\prime} y_{4}^{\prime}=-y_{4}^{\prime} y_{3}^{\prime}, \quad y_{3}^{\prime} y_{i}^{\prime}=y_{i}^{\prime} y_{3}^{\prime}, & \text { for any } 5 \leq i \leq n,\end{cases}
$$

We deduce that $Q \simeq \operatorname{Frac}\left(\widetilde{\mathcal{O}}\left(\mathbb{C}^{2}\right) \otimes T_{n-2}\right)$ where $T_{n-2}$ is the subalgebra of $Q$ generated by the elements $\left(y_{i}^{\prime}\right)_{3 \leq i \leq n}$. Applying the same reduction on the generators of $T_{n-2}$, we conclude by iteration and using Proposition 1.1.3 that $Q \simeq \operatorname{Frac}\left(\widetilde{\mathcal{O}}\left(\mathbb{C}^{n}\right)\right)$.

3.2.5. Lemma. For any $1 \leq i \leq n-1$ and any $1 \leq m \leq n$, we have:

$$
\begin{aligned}
{\left[t_{i}, u_{m, i, i+1}\right] } & =x_{m, i}^{2} & \\
{\left[t_{i}, u_{m, k, i+1}\right] } & =u_{m, k, i} & \text { for } m \leq k<i \leq n, \\
{\left[t_{i}, u_{m, i+1, \ell}\right] } & =u_{m, i, \ell} & \text { for } m<i+1<\ell \leq n, \\
{\left[t_{i}, u_{m, i+1, \ell}\right] } & =0 & \text { for } m=i+1<\ell \leq n, \\
{\left[t_{i}, u_{m, k, \ell}\right] } & =0 & \text { for } k \neq i+1 \text { and } \ell \neq i+1, \\
{\left[t_{i}, x_{m, i+1}\right] } & =x_{m, i} & \text { for } i+1>m, \\
{\left[t_{i}, x_{m, i+1}\right] } & =0 & \text { for } i+1=m, \\
{\left[t_{i}, x_{m, k}\right] } & =0 & \text { for } k \neq i+1 .
\end{aligned}
$$

Proof. We study the action on $B$ of the Chevalley generators $t_{1}, t_{2}, \ldots, t_{n-1}$ defined in 2.2. Let us recall that, by (38) we have:

$$
\left[t_{i}, b_{i+1}^{+}\right]=b_{i}^{+} \quad \text { and }\left[t_{i}, b_{k}^{+}\right]=0 \text { if } k \neq i+1 \text {. }
$$

Similarly by (23) to (27), we obtain:

$$
\begin{array}{rlrl}
{\left[t_{i}, a_{i, i+1}^{+}\right]} & =\left(b_{i}^{+}\right)^{2}, & \\
{\left[t_{i}, a_{k, i+1}^{+}\right]} & =a_{k, i}^{+} & \text {for } 1 \leq k<i \leq n, \\
{\left[t_{i}, a_{i+1, \ell}^{+}\right]} & =a_{i, \ell}^{+} & \text {for } i+1<\ell \leq n, \\
{\left[t_{i}, a_{k, \ell}^{+}\right]} & =0 \quad \text { for } k \neq i+1 \text { and } \ell \neq i+1 .
\end{array}
$$

Then for fixed $i$, we prove the assertions of the lemma by induction on $m$. By (54), assertion (71) is the case $m=1$ of assertions (68) to (70), and (72) to $(75)$ are the case $m=1$ of assertions $(63)$ to $(67)$. Suppose that the eight assertions are satisfied to some rank $m \geq 1$. By (55), we have:

$$
\begin{aligned}
{\left[t_{i}, u_{m+1, k, \ell}\right]=\left[t_{i}, u_{m, m, k}\right] } & u_{m, m, \ell}+u_{m, m, k}\left[t_{i}, u_{m, m, \ell}\right] \\
& -\left[t_{i}, u_{m, k, \ell}\right] x_{m, m}^{2}-u_{m, k, \ell}\left[t_{i}, x_{m, m}^{2}\right] .
\end{aligned}
$$

It follows from (69) and (70) that the fourth term is zero. Then using induction assumptions (63) to (67), tedious but straightforward calculations prove that these five assertions remain valid at the rank $m+1$. Similarly by (55) we have:

$$
\left[t_{i}, x_{m+1, k}\right]=\left[t_{i}, x_{m, m}\right] x_{m, k}+x_{m, m}\left[t_{i}, x_{m, k}\right]-\left[t_{i}, u_{m, m, k}\right] .
$$

It follows from $(69)$ and $(70)$ that the first term is zero. Then using induction assumptions (63) to (70), it is easy to check that: $\left[t_{i}, x_{m+1, i+1}\right]$ equals 0 if 
$m+1=i+1$ and equals $x_{m+1, i}$ if $i+1>m+1$, and $\left[t_{i}, x_{m+1, k}\right]=0$ for any $k \neq i+1$. This finishes the proof.

3.2.6. Corollary. Each generator $t_{i j}$ for $1 \leq i<j \leq n$ commutes with each generator $y_{m}$ for $1 \leq m \leq n$.

Proof. By (69) and (70), the Chevalley generators $t_{1}, t_{2}, \ldots, t_{n-1}$ commute with $y_{m}=x_{m, m}$ for any $1 \leq m \leq n$. By (28) and (36), each generator $t_{i j}$ for $1 \leq i<j \leq n$ is an iterated bracket of $t_{1}, t_{2}, \ldots, t_{n-1}$.

3.2.7. Proposition. The skewfield of fractions of $U^{+}$satisfies:

$$
F=Q\left(a_{i j}^{+}\right)_{1 \leq i<j \leq n}\left(t_{i j} ; d_{i j}\right)_{1 \leq i<j \leq n},
$$

where the generators $t_{i j}$ commute with all elements of $Q$, and the derivations $d_{i j}$ translate the commutation relations between the $t_{i j}$ 's and the $a_{i j}^{+}$'s [see relations (23) to (27)] or the $t_{i j}$ 's with each other [see relations (28) to (30)].

Proof. This is just a summary of assertions (53) and (60), Proposition 3.2.4 and Corollary 3.2.6.

3.2.8. Lemma. Denote by $\left(q_{i, j}\right)_{1 \leq i<j \leq n}$ the elements of $F$ defined by:

$$
q_{i, j}:=y_{i}^{-2} u_{i, i, j} \quad \text { for any } 1 \leq i<j \leq n .
$$

Then the $q_{i, j}$ 's commute with each other and with all elements of $Q$, and the subfield of $L$ generated over $Q$ by the $\left(q_{i, j}\right)_{1 \leq i<j \leq n}$ is equal to $L$.

Proof. The first assertion follows from Corollary 3.2.3 and third relation in 59. To prove the second assertion, we observe directly from definition 55 that for any $1 \leq i<j \leq n$, we have:

$$
u_{i, i, j}=e_{i-1, j}+(-1)^{i-1} a_{i, j}^{+} y_{1}^{2} y_{2}^{2} \cdots y_{i-1}^{2},
$$

where the element $e_{i-1, j}$ lies in the subalgebra $E_{i-1}$ generated by $y_{1}^{2}, y_{2}^{2}, \ldots, y_{i-1}^{2}$ and the $a_{k, \ell}^{+}$'s for $1 \leq k \leq i-1$. Then we can write:

$$
a_{i, j}^{+}=(-1)^{i-1} y_{1}^{-2} y_{2}^{-2} \cdots y_{i-1}^{-2} q_{i, j}+e_{i-1, j}^{\prime}
$$

with $e_{i-1, j}^{\prime}:=(-1)^{i} y_{1}^{-2} y_{2}^{-2} \cdots y_{i-1}^{-2} e_{i-1, j} \in E_{i-1}$. This proves the rest of the lemma by induction on $i$.

3.2.9. Lemma. For any $1 \leq i<j \leq n$ and any $1 \leq k<\ell \leq n$, we have:

$$
\begin{aligned}
& {\left[t_{i j}, q_{i, j}\right]=1 \text {, }} \\
& {\left[t_{i j}, q_{k, \ell}\right]=0 \quad \text { if } j \neq \ell,} \\
& {\left[t_{i j}, q_{k, j}\right]=0 \quad \text { if } i<k \text {, }} \\
& {\left[t_{i j}, q_{k, j}\right]=q_{k, i} \quad \text { if } i>k .}
\end{aligned}
$$

Proof. We proceed by induction on $j-i$. For $j=i+1$, the identities directly follows from Lemma 3.2.5. For the general case, we deduced from (28) and (34) that $t_{i, j+1}=\left[t_{i, j}, t_{j, j+1}\right]=\left[t_{i, j}, t_{j}\right]$. Then we have $\left[t_{i, j+1}, q_{k, \ell}\right]=$ $\left[\left[t_{i, j}, t_{j}\right], q_{k, \ell}\right]=\left[t_{i, j},\left[t_{j}, q_{k, \ell}\right]\right]-\left[t_{j},\left[t_{i, j}, q_{k, \ell}\right]\right]$ for any $1 \leq k<\ell \leq n$, and we 
finish the proof by induction considering successively the different cases for the indices $(i, j)$ and $(k, \ell)$.

3.2.10. Corollary. The elements $\left(q_{i, j}\right)_{1 \leq i<j \leq n}$ defined in Lemma 3.2 .8 and the elements $\left(p_{i, j}\right)_{1 \leq i<j \leq n}$ defined inductively by:

$$
p_{1, j}:=t_{1, j} \quad \text { and } \quad p_{i, j}:=t_{i, j}-\sum_{m=1}^{i-1} q_{m, i} p_{m, j}
$$

satisfy $\left[p_{i j}, q_{i j}\right]=1$ and $\left[p_{i j}, p_{k \ell}\right]=\left[q_{i j}, q_{k \ell}\right]=\left[p_{i j}, q_{k \ell}\right]=0$ for $(i, j) \neq(k, \ell)$.

Proof. Follows obviously from Lemma 3.2 .9 by induction on $i$.

3.2.11. Proposition. We have: $F=Q\left(q_{i j}\right)_{1 \leq i<j \leq n}\left(p_{i j} ; \partial_{q_{i j}}\right)_{1 \leq i<j \leq n}$.

Proof. It's clear by Lemma 3.2 .8 that $L=Q\left(q_{i j}\right)_{1 \leq i<j \leq n}$. By definition 82 and Proposition 3.2.7, the subfield of $F$ generated over $L$ by the elements $\left(p_{i, j}\right)_{1 \leq i<j \leq n}$ is equal to $F$. Hence the proposition just summaries the results of Lemma 3.2.8 and Corollary 3.2.10.

3.2.12. Corollary. The skewfield $F$ is isomorphic to Frac $\left(A_{n(n-1) / 2} \otimes \widetilde{\mathcal{O}}\left(\mathbb{C}^{n}\right)\right)$, and its center is $\mathcal{Z}(F)=\mathcal{Z}(Q)=\mathbb{C}\left(y_{1}^{2}, \ldots, y_{n}^{2}\right)$.

Proof. By Corollary 3.2.10, the generators $p_{i j}$ and $q_{i j}$ commute with all elements of $Q$ and the subalgebra $W$ of $F$ they generate is isomorphic to the

usual Weyl algebra $A_{n(n-1) / 2}$. Then the theorem follows from Proposition 3.2.11 and assertion 61) of Proposition 3.2.4.

3.2.13. Conclusion. We have proved the first assertion of the main theorem.

\subsection{Enveloping skewfield of the Lie subalgebra $\mathfrak{n}_{\overline{0}}^{+}$.}

3.3.1. Notations. In order to determine the skewfield of fractions of the enveloping algebra of the even part $\mathfrak{n}_{0}^{+}$of $\mathfrak{n}^{+}$described in (48), we complete the notations (51), (52), (53) and (61) by defining:

$$
\begin{aligned}
Q_{0} & :=\mathbb{C}\left(y_{1}^{2}, y_{2}^{2}, \ldots, y_{n}^{2}\right) \subset Q, \\
L_{0} & :=K\left(\left(b_{1}^{+}\right)^{2},\left(b_{2}^{+}\right)^{2}, \ldots,\left(b_{n}^{+}\right)^{2}\right) \subset L, \\
F_{0} & :=\operatorname{Frac} \mathcal{U}\left(\mathfrak{n}_{0}^{+}\right)=L_{0}\left(t_{i j} ; d_{i j}\right)_{1 \leq i<j \leq n} \subset F .
\end{aligned}
$$

3.3.2. Lemma. We have the equality $L_{0}=K\left(y_{1}^{2}, y_{2}^{2}, \ldots, y_{n}^{2}\right)$.

Proof. Returning to notations (55) and using (59) and (58), we check by straightforward calculations that: $x_{k, i}^{2}=-x_{k-1, k-1}^{2} x_{k-1, i}^{2}+u_{k-1, k-1, i}^{2}$ for all $2 \leq k \leq i \leq n$. Using this identity and point (ii) of Lemma 3.2 .3 , we prove that $y_{1}^{2}=\left(b_{1}^{+}\right)^{2}, y_{2}^{2}=-\left(b_{1}^{+}\right)^{2}\left(b_{2}^{+}\right)^{2}+a_{1,2}^{2}$, and :

$$
y_{i}^{2}=(-1)^{i-1} y_{1}^{2} y_{2}^{2} \cdots y_{i-1}^{2}\left(b_{i}^{+}\right)^{2}+v_{i-1} \text { for any } 2 \leq i \leq n,
$$

where the term $v_{i-1}$ lies in the subalgebra generated over $A$ by $y_{1}^{2}, y_{2}^{2}, \ldots, y_{i-1}^{2}$. Then the result follows by induction on $i$. 
3.3.3. Proposition. $F_{0}=Q_{0}\left(q_{i j}\right)_{1 \leq i<j \leq n}\left(p_{i j} ; \partial_{q_{i j}}\right)_{1 \leq i<j \leq n}$, with center $Q_{0}$.

Proof. It is clear by previous lemma that $L_{0}=Q_{0}\left(a_{i j}\right)_{1 \leq i<j \leq n}$. Then the calculations in the proof of Lemma 3.2 .8 show that the $q_{i, j}$ 's are elements of $L_{0}$, and moreover that the subfield of $L_{0}$ generated over $Q_{0}$ by $\left(q_{i j}\right)_{1 \leq i<j \leq n}$ is equal to $L_{0}$. Finally we deduce from (82) that the skew subfield of $F_{0}$ generated over $L_{0}$ by $\left(p_{i j}\right)_{1 \leq i<j \leq n}$ is equal to $F_{0}$.

3.3.4. Conclusion. The previous proposition showed that:

$$
\operatorname{Frac} \mathcal{U}\left(\mathfrak{n}_{0}^{+}\right) \simeq \operatorname{Frac}\left(A_{n(n-1) / 2}(\mathbb{C}) \otimes \mathcal{O}\left(\mathbb{C}^{n}\right)\right)
$$

Hence we retrieve in the context of the classical Gelfand-Kirillov property the usual description of the enveloping skewfield of the nilpotent Lie algebra $\mathfrak{s p}^{+}(2 n)$ as a Weyl skewfield $D_{n(n-1) / 2}$ over a purely transcendental extension of dimension $n$ over $\mathbb{C}$.

3.4. Enveloping skewfield of the Lie subsuperalgebra $\mathfrak{b}^{+}$. Resuming all notations of the previous section and using (49) and (53), we set:

$$
H:=\operatorname{Frac} U_{b}^{+}=F\left(k_{1}, \Delta_{1}\right)\left(k_{2}, \Delta_{2}\right) \cdots\left(k_{n}, \Delta_{n}\right) .
$$

Our first step is to determine the action of the generators $k_{1}, \ldots, k_{n}$ of the Cartan subalgebra $\mathfrak{h}$ on the generators $y_{i}, p_{i j}, q_{i j}(1 \leq i<j \leq n)$ introduced in the description of $F$ in Proposition 3.2.11.

3.4.1. Lemma. For any $1 \leq \ell \leq n$, we have the following identities:

$$
\begin{aligned}
& {\left[k_{\ell}, y_{m}\right]=\left\{\begin{array}{ll}
y_{\ell} & \text { if } m=\ell, \\
2^{m-\ell-1} y_{m} & \text { if } m>\ell,
\end{array} \text { and }\left[k_{\ell}, y_{m}\right]=0 \text { if } m<\ell .\right.} \\
& \left\{\begin{array}{lll}
{\left[k_{\ell}, q_{\ell, j}\right]=-q_{\ell, j}} & {\left[k_{\ell}, p_{\ell, j}\right]=p_{\ell, j}} & \text { for any } j>\ell, \\
{\left[k_{\ell}, q_{i, \ell}\right]=q_{i, \ell}} & {\left[k_{\ell}, p_{i, \ell}\right]=-p_{i, \ell}} & \text { for any } i<\ell, \\
{\left[k_{\ell}, q_{i, j}\right]=0} & {\left[k_{\ell}, p_{i, j}\right]=0} & \text { for any } i \neq \ell \neq j .
\end{array}\right.
\end{aligned}
$$

Proof. We start with the action of $k_{\ell}$ on $b_{i}^{+}$and $a_{i, j}^{+}$for any $1 \leq i<j \leq n$ given by relations (32) and (33). Using the iterative definitions (54) and (55), we obtain for $1 \leq m<\ell$ the identities:

$$
\left[k_{\ell}, x_{m, i}\right]=\left\{\begin{array}{ll}
x_{m, \ell} & \text { if } i=\ell, \\
0 & \text { if } i \neq \ell,
\end{array} \text { and }\left[k_{\ell}, u_{m, i, j}\right]= \begin{cases}u_{m, \ell, j} & \text { if } i=\ell, \\
u_{m, i, \ell} & \text { if } j=\ell, \\
0 & \text { if } i \neq \ell \neq j .\end{cases}\right.
$$

Similarly for $m=\ell$ :

$$
\left[k_{\ell}, x_{\ell, i}\right]=\left\{\begin{array}{ll}
x_{\ell, \ell} & \text { if } i=\ell, \\
0 & \text { if } i \neq \ell,
\end{array} \text { and }\left[k_{\ell}, u_{\ell, i, j}\right]= \begin{cases}u_{\ell, \ell, j} & \text { if } i=\ell, \\
0 & \text { if } i \neq \ell \neq j .\end{cases}\right.
$$


Finally for $m>\ell$ : $\left[k_{\ell}, x_{m, i}\right]=2^{m-\ell-1} x_{m, i}$ and $\quad\left[k_{\ell}, u_{m, i, j}\right]=2^{m-\ell} u_{m, i, j}$. Assertion (87) follows trivially from these relations since $y_{m}=x_{m, m}$. Moreover, for $1 \leq i, j \leq n$, we have by definition $(77)$ and $(82)$ :

$$
\begin{aligned}
& {\left[k_{\ell}, q_{i, j}\right]=\left[k_{\ell}, y_{i}^{-2} u_{i, i, j}\right]=-2 y_{i}^{-3}\left[k_{\ell}, y_{i}\right] u_{i, i, j}+y_{i}^{-2}\left[k_{\ell}, u_{i, i, j}\right],} \\
& {\left[k_{\ell}, p_{i, j}\right]=\left[k_{\ell}, t_{i, j}\right]-\sum_{m=1}^{i-1}\left[k_{\ell}, q_{m, i}\right] p_{m, j}-\sum_{m=1}^{i-1} q_{m, i}\left[k_{\ell}, p_{m, j}\right] .}
\end{aligned}
$$

We deduce (88) applying case by case the previous identities.

3.4.2. Notation. An obvious observation arising from 88 is:

$$
\left[k_{\ell}, p_{i, j} q_{i, j}\right]=0 \quad \text { for all } 1 \leq \ell \leq n, 1 \leq i<j \leq n .
$$

These relations lead us to introduce the following elements of $H$ :

$$
h_{\ell}:=k_{\ell}+\sum_{m=\ell+1}^{n} q_{\ell, m} p_{\ell, m}-\sum_{m=1}^{\ell-1} q_{m, \ell} p_{m, \ell} \quad \text { for any } 1 \leq \ell \leq n,
$$

which satisfy the relations described in the following lemma.

3.4.3. Lemma. For all $1 \leq \ell, m \leq n$ and $1 \leq i<j \leq n$, we have:

$$
\left[h_{\ell}, q_{i, j}\right]=\left[k_{\ell}, p_{i, j}\right]=\left[h_{\ell}, h_{m}\right]=0 \text { and }\left[h_{\ell}, y_{m}\right]=\left[k_{\ell}, y_{m}\right] \text {. }
$$

Proof. By (92) we have:

$$
\left[h_{\ell}, q_{i, j}\right]=\left[k_{\ell}, q_{i, j}\right]+\sum_{m=\ell+1}^{n} q_{\ell, m}\left[p_{\ell, m}, q_{i, j}\right]-\sum_{m=1}^{\ell-1} q_{m, \ell}\left[p_{m, \ell}, q_{i, j}\right] .
$$

If $i \neq \ell$ and $j \neq \ell$, all brackets appearing in the right hand are zero by Corollary 3.2.10 and relation (88). If $i=\ell$, the equality becomes $\left[h_{\ell}, q_{\ell, j}\right]=\left[k_{\ell}, q_{\ell, j}\right]+q_{\ell, j}\left[p_{\ell, j}, q_{\ell, j}\right]=0$. If $j=\ell$, we obtain $\left[h_{\ell}, q_{i, \ell}\right]=$ $\left[k_{\ell}, q_{i, \ell}\right]-q_{i, \ell}\left[p_{i, \ell}, q_{i, \ell}\right]=0$. The calculations are similar for $p_{i, j}$. It is trivial that $\left[\left(p_{i, j} q_{i, j}\right),\left(p_{i^{\prime}, j^{\prime}} q_{i^{\prime}, j^{\prime}}\right)\right]=0$ for any $1 \leq i<j \leq n$ and $1 \leq i^{\prime}<j^{\prime} \leq n$. Then $\left[h_{\ell}, h_{m}\right]=0$ follows from the commutativity of the Cartan subalgebra and observation (91). By Proposition 3.2.11, the generators $y_{1}, \ldots, y_{n}$ commute with all $p_{i, j}$ 's and $q_{i, j}$ 's; then the last identity of the lemma is clear.

3.4.4. Proposition. Let $W$ be the subalgebra of $F$ generated over $\mathbb{C}$ by the $n(n-1)$ elements $p_{i j}$ and $q_{i j}$. Let $P$ be the subalgebra of $H$ generated over $\mathbb{C}$ by $y_{1}, \ldots, y_{n}$ and $h_{1}, \ldots, h_{n}$. Then $H \simeq \operatorname{Frac}(W \otimes P)$.

Proof. It is clear by (86), (92) and Proposition 3.2.11 that the subfield of $H$ generated over $F$ by $h_{1}, \ldots, h_{n}$ is equal to $H$. Then the result follows from Lemma 3.4.3 and Proposition 3.2.11.

3.4.5. Proposition. Let us define $y_{1}^{\prime}, \ldots, y_{n}^{\prime}$ in $Q$ by:

$$
y_{1}^{\prime}=y_{1} \quad \text { and } \quad y_{m}^{\prime}=y_{1}^{-1} y_{2}^{-1} \cdots y_{m-1}^{-1} y_{m} \quad \text { for any } 2 \leq m \leq n .
$$

Let $P^{\prime}$ be the subalgebra of $H$ generated over $\mathbb{C}$ by $y_{1}^{\prime}, \ldots, y_{n}^{\prime}$ and $h_{1}, \ldots, h_{n}$. Then $P^{\prime} \simeq \widetilde{U}_{n}(\mathbb{C})$ and $H \simeq \operatorname{Frac}\left(W \otimes P^{\prime}\right)$. 
Proof. It is clear by (93) that Frac $P^{\prime} \simeq \operatorname{Frac} P$ and $H \simeq \operatorname{Frac}\left(W \otimes P^{\prime}\right)$. By tedious but simple calculations that we leave to the reader, we have:

$$
y_{\ell}^{\prime} y_{m}^{\prime}=-y_{m}^{\prime} y_{\ell}^{\prime}, \quad\left[h_{\ell}, y_{m}^{\prime}\right]=0 \text { and }\left[h_{\ell}, y_{\ell}^{\prime}\right]=y_{\ell}^{\prime}
$$

for all $1 \leq \ell \neq m \leq n$. We conclude with point (ii) of 1.2 .1 .

3.4.6. Corollary. We have $H \simeq \operatorname{Frac}\left(A_{n(n-1) / 2}(\mathbb{C}) \otimes \widetilde{A}_{n}(\mathbb{C})\right)$, with center $\mathbb{C}$.

Proof. We have already observed in Corollary 3.2 .12 that $W \simeq A_{n(n-1) / 2}(\mathbb{C})$ and in Remark 1.2.4 that Frac $\widetilde{A}_{n}(\mathbb{C}) \simeq \operatorname{Frac} \widetilde{U}_{n}(\mathbb{C})$. Then the isomorphism $H \simeq \operatorname{Frac}\left(A_{n(n-1) / 2}(\mathbb{C}) \otimes \widetilde{A}_{n}(\mathbb{C})\right)$ follows from previous proposition. Using the usual notation $D_{m}(E)$ for the skewfield of fractions of a Weyl algebra $A_{m}(E)$ over a skewfield $E$, we have $H \simeq D_{n(n-1)}(E)$ for $E=\operatorname{Frac} \widetilde{A}_{n}(\mathbb{C})$. Its center satisfies $\mathcal{Z}(H) \simeq \mathcal{Z}\left(D_{n(n-1)}(E)\right)=\mathcal{Z}(E)$, and $\mathcal{Z}(E)=\mathbb{C}$ by Proposition 3.3.1 of [18].

3.4.7. Conclusion. The second assertion of the main theorem is proved.

\subsection{Enveloping skewfield of the Lie subalgebra $\mathfrak{b}_{\overline{0}}^{+}$.}

3.5.1. Notations. In order to determine the skewfield of fractions of the enveloping algebra of the even part $\mathfrak{b}_{\overline{0}}^{+}$of $\mathfrak{b}^{+}$described in (50), we denote:

$$
H_{0}:=\operatorname{Frac} \mathcal{U}\left(\mathfrak{b}_{\overline{0}}^{+}\right)=F_{0}\left(k_{1} ; \Delta_{1}\right) \cdots\left(k_{n} ; \Delta_{n}\right),
$$

where $F_{0}$ is described by Proposition 3.3 .3 .

3.5.2. Proposition. We have $H_{0} \simeq$ Frac $\left(W \otimes P_{0}^{\prime}\right)$, where $P_{0}^{\prime}$ is the subalgebra of $P^{\prime}$ generated by $\left(y_{1}^{\prime}\right)^{2}, \ldots,\left(y_{n}^{\prime}\right)^{2}, h_{1}, \ldots, h_{n}$, which is isomorphic to the enveloping algebra $U_{n}(\mathbb{C})$ defined in point (i) of 1.2.1.

Proof. By Proposition 3.3 .3 and $(92)$, the subfield of $H_{0}$ generated over $F_{0}$ by $h_{1}, \ldots, h_{n}$ is equal to $H_{0}$. Since Frac $P_{0}^{\prime}=Q_{0}$ by (93), the result follows from Proposition 3.3.3.

3.5.3. Conclusion. Because of the isomorphism Frac $U_{n}(\mathbb{C}) \simeq \operatorname{Frac} A_{n}(\mathbb{C})$ observed in Remark 1.2.4 and the canonical isomorphism $A_{n(n+1) / 2}(\mathbb{C}) \simeq$ $A_{n(n-1) / 2}(\mathbb{C}) \otimes A_{n}(\mathbb{C})$, the previous proposition proves that:

$$
\operatorname{Frac} \mathcal{U}\left(\mathfrak{b}_{\overline{0}}^{+}\right) \simeq \operatorname{Frac} A_{n(n+1) / 2}(\mathbb{C}) .
$$

Then we retrieve in the context of classical Gelfand Kirillov property the usual description of the enveloping skewfield of the solvable Borel subalgebra of the Lie algebra $\mathfrak{s p}(2 n)$ as the Weyl skewfield $D_{n(n+1) / 2}(\mathbb{C})$.

3.6. Final observation. A thorough reading of the previous proofs shows that the isomorphisms we obtain at the level of the skewfields of fractions are already realized at the level of the localization of the algebras under consideration by the multiplicative set generated by the $n$ elements $y_{1}, \ldots, y_{n}$. Since these elements are homogeneous, this result is consistent with the notion of skewfield of fractions in the category of superalgebras. 


\section{REFERENCES}

[1] J. Alev and F. Dumas, Sur le corps de fractions de certaines algèbres quantiques. J. Algebra 170 (1) (1994) 229-265.

[2] J. Alev and F. Dumas, On enveloping skew fields of some Lie superalgebras. J. Algebra Appl. 15 (2016) 1650071, 16 pp.

[3] J. Alev, A. Ooms and M. Van Den Bergh, The Gelfand-Kirillov conjecture for Lie algebras of dimension at most eight. J. Algebra 227 (2) (2000) 549-581.

[4] M. Aubry and J.-M. Lemaire, Zero divisors in enveloping algebras of graded Lie algebras. J. Pure Appl. Algebra 38 (2-3) (1985) 159-166.

[5] W. Borho, P. Gabriel and R. Rentschler, Primideale in Einhüllenden auflösbarer LieAlgebren, Lecture Notes in Mathematics, 357, Springer-Verlag, Berlin-New York, 1973.

[6] A. Ch. Ganchev and T. D. Palev, A Lie superalgebraic interpretation of the paraBose statistics. J. Math. Phys. 21 (4) (1980) 797-799.

[7] I. M. Gelfand and A. A. Kirillov, Sur les corps liés aux algèbres enveloppantes des algèbres de Lie, Inst. Hautes Etudes Sci. Publ. Math. 31 (1966) 5-19.

[8] A. Giaquinto and J. J. Zhang, Quantum Weyl algebras. J. Algebra 176 (3) (1995) 861-881.

[9] M. Gorelik and E. Lanzmann, The annihilation theorem for the Lie superalgebra osp (1,2l). C. R. Acad. Sci. Paris 327 (3) (1998) 237-242.

[10] A. Joseph, Proof of the Gelfand-Kirillov conjecture for solvable Lie algebras, Proc. Amer. Math. Soc. 45 (1974), 1-10.

[11] V. G. Kac, Lie superalgebras. Advances in Math. 26 (1) (1977) 8-96.

[12] J. C. McConnell, Representations of solvable Lie algebras and the Gelfand-Kirillov conjecture, Proc. London Math. Soc. 29 (1974), 453-484.

[13] I. M. Musson, On the Goldie quotient ring of the enveloping algebra of a classical simple Lie superalgebra. J. Algebra 235 (1) (2001) 203-213.

[14] I. M. Musson, Lie superalgebras and enveloping algebras. Graduate Studies in Mathematics, 131. American Mathematical Society, Providence, RI, 2012. xx+488 pp.

[15] T. D. Palev and J. Van der Jeugt, The quantum superalgebra $U_{q}[\operatorname{osp}(1 \mid 2 n)]$ : deformed para-Bose operators and root of unity representations. J. Phys. A 28 (9) (1995) 2605-2616.

[16] A. Premet, Modular Lie algebras and the Gelfand-Kirillov conjecture, Invent. Math. 181 (2) (2010) 395-420.

[17] L. Richard, Hochschild homology and cohomology of some classical and quantum noncommutative polynomial algebras. J. Pure Applied. Algebra 187 (1-3) (2004) 255-294.

[18] L. Richard, Équivalence rationnelle d'algèbres polynomiales classiques et quantiques. J. Algebra 287 (1) (2005) 52-87.

[19] M. Wambst, Complexes de Koszul quantiques. Ann. Inst. Fourier (Grenoble) 43 (4) (1993), 1089-1156.

(J. Alev) Université de Reims, Laboratoire de Mathématiques (FRE 2011 - CNRS), Moulin de la Housse, B.P. 1039, 51687 Reims Cedex 2 (France)

E-mail address: jacques.alev@univ-reims.fr

(F. Dumas) Université Clermont Auvergne, Laboratoire de Mathématiques Blaise Pascal (UMR 6620 - CNRS), 3 Place Vasarely, CS 60026, 63178 Aubière CEDEX (FRANCE)

E-mail address: Francois.Dumas@uca.fr 